\title{
Dynamic Risk Analysis on City Waterlogging Disaster Based on Scenario Simulation
}

\author{
Xilei Pang ${ }^{1}$, Chongfu Huang ${ }^{2}$, Yingju Zhang ${ }^{3}$ \\ ${ }^{1}$ Shenzhen MINTAIAN Risk Management Research Institute, Shenzhen 518034 \\ ${ }^{2}$ Academy of Disaster Reduction and Emergency Management, Beijing Normal University, Beijing 100075 \\ ${ }^{3}$ Dalian Administrative College, Dalian 116013
}

\section{基于情景的城市内港灾害动态风险分析研究}

\author{
庞西磊 ${ }^{1}$, 黄崇福 ${ }^{2}$, 张英菊 ${ }^{3}$ \\ '深圳市民太安风险管理研究院, 广东 深圳 518034 \\ ${ }^{2}$ 北京师范大学（民政部/教育部）减灾与应急管理研究院，北京 100075 \\ ${ }^{3}$ 大连行政学院, 辽宁 大连 110613
}

\begin{abstract}
In the view of systematicness and uncertainty of disaster risk, the paper firstly adopts parameter estimation statistical algorithm, information diffusion model and geographic information space analysis technology to analyze hazard occurrence possibility, affected population vulnerability and rainstorm disaster simulation according to the data we have. Then, it uses the comprehensive risk assessment to calculate the dynamic risk year by year. Finally, it gives dynamic risk scenarios for the city waterlogging disaster in different rainfall conditions in the coming five years (2016-2020).
\end{abstract}

Keywords: City waterlogging, Dynamic risk, Hazard, Disaster bearing-body, Scenario

\section{摘要}

本文从灾害风险的系统性和不确定性出发, 根据致灾 因子和承灾体数据样本的特征, 首先采用参数估计统 计算法、信息扩散模型和地理信息空间分析技术，分 别开展了城市内涝灾害致灾因子的可能性计算、受灾 人口的脆弱性评估和不同降雨情景下的内涝积水和 受灾人口分布灾情模拟; 然后利用综合风险评估的基 本模式逐年耦合计算灾害风险; 最终给出了案例区未
来五年（2016-2020）在不同降雨条件下的城市内涝 灾害动态风险情景的变化情况。

关键词: 城市内涝, 动态风险, 致灾因子, 承灾体, 情景

\section{1. 研究背景}

洪涝灾害是许多国家和地区面临的主要自然灾 害之一。由于全球气候变化引起的区域性气候波动, 导致极端暴雨天气频繁出现, 因暴雨造成的洪涝灾害 发生频次已经超过台风、地震、泥石流、干旱等自然 灾害, 有分析报告通过对 2001-2010 十年间全球爆发 的四千多起巨灾中分析发现, 洪涝灾害占比例最大; 该报告统计数据还显示, 中国近十年来的洪涝灾害造 成的受灾人口和经济损失规模均列世界首位 ${ }^{[1]}$ 。而这 些洪涝灾害有很大一部份都发生在人口集中和经济 发展迅速的城镇地区。

由于城市人口密集、财产集中, 洪涝灾害经常造 成大面积险情。为了减少洪涝灾害对生命和财产安全 的威胁, 人们通过不断努力展开了卓有成效的洪港灾 害研究。例如: 有研究在地理信息系统平台上将降雨 径流的水文模型、排水系统的一维水动力模型和地表 洪水漫流的二维水动力模型进行耦合, 建立了适用于 城市洪水预测模拟的耦合模型系统 ${ }^{[2]}$; 有学者在修正 已有的城市高程模型基础上, 利用 GIS 柱格空间分 析技术模拟了不同重现期条件下的淹没深度和范围， 并以街道为研究单元对上海浦东新区进行了暴雨内 
Risk Analysis and Crisis Response in Big Data Era (RAC-16)

涝危险性评价 ${ }^{[3]}$ 。另有研究采用模糊综合评判、模糊 分类和模糊近似度相结合的方法, 对马来西亚科拉坦 地区进行了模糊意义上的洪灾风险区划图 ${ }^{[4]}$ 。也有研 究者通过对土地利用经济赋值、分析人类活动暴露性 绘制了全球暴露分布图, 然后结合基于 GIS 制成的 洪涝致灾图进行重新秚释和分类, 最后给出了小流域 的洪港灾害风险图 ${ }^{[5]}$ 。有学者基于大型的标准化地理 信息数据库, 通过对住房、其他类型建筑、室内财产、 车辆和个人等承载体暴露性和结果分析, 模拟了不同 极端事件下的区域损失情景 ${ }^{[6]}$ 。另有研究选择城市暴 雨内涝灾害为研究对象, 提出了一套基于小尺度的城 市暴雨内涝灾害风险评估的思路与方法 ${ }^{[7]}$ 。还有研究 从宏观角度, 考虑到人类认知能力的提高带来的信息 量增长、自然系统变化造成的不确定性增多、自然与 人类社会系统之间的正负反馈等因素, 提出了基于进 化 33 系统论的洪涝灾害风险宏观分析框架 ${ }^{[8]}$ 。

由上可知, 当前城市洪涝灾害风险研究主要集中 在水动力模型研究、致灾因子危险性分析、承载体的 脆弱性和易损性评价等方面, 这些研究利用了暴雨洪 涝形成的物理数学模型和计算机仿真模拟技术, 从不
同角度对城市洪涝灾害以及城市洪涝灾害风险进行 了不同层次的探讨和研究。

本文借鉴综合风险评估的基本模式 ${ }^{[9]}$, 并借鉴相 关研究理论, 展开城市内涝灾害动态风险分析的探索 性研究, 希望可以为政府进行灾害应急管理、制定防 灾减灾规划、以及保险公司制定保险方案等工作提供 参考意见和技术支撑。

\section{2. 研究方法与数据准备}

\section{1. 技术路线}

本文首先利用案例区 (汕头市龙湖区) 的降雨数 据, 制作出降雨日、月、年极值序列样本, 并统计降 雨量的年月变化和不同历时的最大降雨量, 给出案例 区的降雨分布概率模型; 然后, 利用人口动态预测模 型和灾害仿真模拟系统, 开展暴雨内涝情景模拟和承 灾体脆弱性分析; 最后, 进行风险耦合和对比, 结合 受灾人口分布情景图, 给出案例区在未来某时段内的 动态风险分析结果。技术路线图如下:

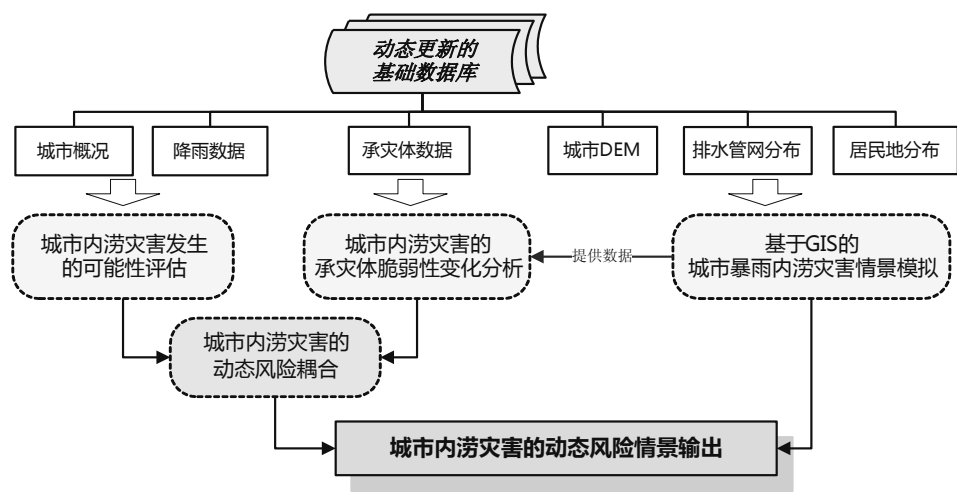

图 1 城市内涝灾害的动态风险分析流程

\section{2. 数据准备}

用于开展内涝灾害动态风险分析的数据主要包 括以下几个方面:

(1) 城市基础地理信息数据

城市基础地理信息数据主要包括: 城市地形数字 高程 (空间差值)、居民地分布、城市排水管网的分 布 (模拟数据) 等。

(2) 致灾因子基础数据

致灾因子数据即案例区 1951-2013 年间的降雨数 据 ${ }^{[10]}$, 经过篮选整理, 采用以年为基本单位的连续时
间序列降雨极值数据, 用于拟合致灾因子的概率分布 模型。

(3) 承灾体基础数据

案例区的历史人口统计数据来自汕头市统计年 鉴; 受灾人口通过灾害模拟系统的模拟计算得到。

\section{3. 城市内涝灾害发生的可能性评估}

通过年和月降雨量的统计分析发现, 汕头市的年 度最大月降雨量主要集中在夏季的 5 8 月。很明显, 暴雨是该城市发生内涝灾害的主要致灾因子。 
Risk Analysis and Crisis Response in Big Data Era (RAC-16)

通过对案例区气象站点 1951-2013 年期间的降雨 日极值序列数据的收集和整理, 利用 EasyFit 和 Matlab 软件, 对降雨极值数据进行了常见的 20 种连
续函数分布分析（参见表 1 ），并利用 Anderson-Darling(A-D 值)法检验了函数分布模型及 其参数的可靠性 (拟合度)。

表 1 基于 A-D 检验的汕头市降雨极值分布函数类型的拟合度对比表

\begin{tabular}{c|ccccccc}
\hline 分布类型 & Weibull (3P) & Pearson5(3P) & Log-Gamma & Inv. Gaussian & Gamma & Gumbel Max & $\cdots \cdots$ \\
\hline A-D 值 & 0.0923 & 0.1259 & 0.1274 & 0.1282 & 0.1401 & 0.1611 & $\cdots \cdots$ \\
\hline
\end{tabular}

表 2 汕头市暴雨降雨分布函数及其参数对比表

\begin{tabular}{c|c|c|c}
\hline 降雨序列 & 函数分布类型 & 参数变量值 & 拟合度 (A-D 值) \\
\hline \multirow{2}{*}{$1951-2013$} & Weibull(3P) & $\alpha=1.816 \quad \beta=104.73 \quad \gamma=52.879$ & 0.0923 \\
\hline
\end{tabular}

通过对比函数的可靠性, 认为日极值降雨的概率 分布类型属于 Weibull (3P) 型分布 (见表 2)。Weibull (3P) 型分布是指由三个参数变量 $\alpha 、 \beta$ 和 $\gamma$ 约束 的 Weibull 分布函数, 其概率密度函数的基本表达式 是:

$$
f(x)=\frac{\alpha}{\beta}\left(\frac{x-\gamma}{\beta}\right)^{\alpha-1} \exp \left(-\left(\frac{x-\gamma}{\beta}\right)^{\alpha}\right) \text { 其中, } \gamma<x<\infty
$$

（公式 1）

则, 其超越概率分布函数的基本表达式为:

$$
P(x)=\exp \left(-\left(\frac{x-\gamma}{\beta}\right)^{\alpha}\right) \text { 其中, } \gamma<x<\infty （ \text { 公式 2） }
$$

公式 1 和公式 2 中的 $\mathrm{x}$ 表示降雨量、 $\alpha$ 是函数的 形状参数、 $\beta$ 代表尺寸参数、 $\gamma$ 是位置参数。

基于上述分析结果, 将降雨极值分布的超越概率 密度函数转换为曲线图 (见图 2)。如果用 I 代表降雨 量, RP 表示降雨重现期。根据表 1 和公式 2 , 可知 案例区的未来降雨重现期计算公式:

$$
R P=\exp \left(\frac{I-52.879}{104.73}\right)^{1.816}
$$

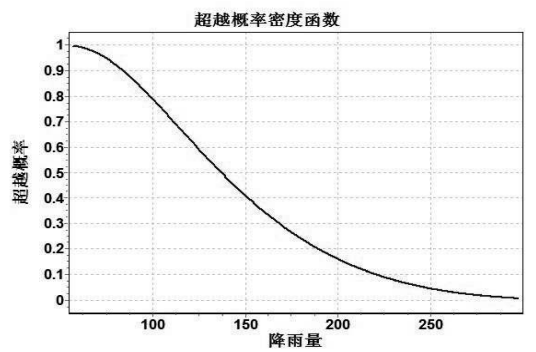

图 2 降雨分布超越概率函数曲线图

考虑到全球气候变化的影响, 在未来不同的温室 气体排放情境下, 包含我国东南沿海地区在内的东亚 地区在不同季节的温度会有小幅上升, 降雨有可能会 增加 ${ }^{[1]}$ (参见表 3 )。未来的气候变化会对区域洪涝 灾害造成明显影响, 导致城市地区特别是某些河口三 角洲地区会面临更多的洪涝灾害风险 ${ }^{[12,13]}$ 。因此, 对 敏感地区进行区域降雨强度分析时, 有必要考虑因气 候变暖可能导致的降雨变化情况。

由历史灾情数据（参见表 4) 可以看出, 案例区 历史发生城市内涝灾害的暴雨日降水量最低值约为 $49 \mathrm{~mm}$, 最高值约为 $147 \mathrm{~mm}$, 我们可以依此作为案例 区内涝灾害模拟的致灾因子输入值的上下限。那么, 结合表 3 和表 4 数据, 可以合理地假设案例区在未来 遭受不同暴雨的极限值 (见表 5)。

表 3 未来不同排放情景下的区域温度和降雨变化预测

\begin{tabular}{c|c|cc|cc}
\hline \multirow{2}{*}{ 影响范围 } & \multicolumn{5}{|c}{2010 年-2039 年 } \\
\cline { 2 - 5 } & 季节 & \multicolumn{2}{|c|}{$\begin{array}{c}\text { 温度上升幅度 }\left({ }^{\circ} \mathrm{C}\right) \\
\mathrm{A} 1 \mathrm{FI}\end{array}$} & $\mathrm{B} 1$ & \multicolumn{2}{|c}{ 降雨量增加幅度 $(\%)$} \\
\hline 东亚 $(20 \mathrm{~N}-50 \mathrm{~N} ; 100 \mathrm{E}-150 \mathrm{E})$ & 夏 & 1.61 & 1.5 & 2 & 2 \\
\hline
\end{tabular}

（注: 表中的 A1FI 代表未来时段的最高排放情景; B1 代表未来时段的最低排放情景) 
Risk Analysis and Crisis Response in Big Data Era (RAC-16)

表 4 案例区的暴雨内涝历史灾情 (受灾人口) 统计

\begin{tabular}{ccc}
\hline 灾害发生日期 & 日降雨量 $(\mathrm{mm})$ & 受灾人口 \\
\hline $1986 / 7 / 11$ & 146.7 & 5263 \\
$1991 / 7 / 19$ & 49.1 & 4929 \\
$2005 / 6 / 21$ & 105.5 & 495500 \\
$2005 / 8 / 13$ & 128.3 & 246100 \\
$2006 / 7 / 15$ & 127.8 & 1062900 \\
\hline
\end{tabular}

表 5 城市暴雨内涝灾害降雨条件模拟的因素选取

\begin{tabular}{c|cc}
\hline 取值 & 修正前的日降雨量 (单位:mm) & 据气候变化影响修正的日降雨量 (单位:mm) \\
\hline 最小值 & 49 & 50 \\
最大值 & 147 & 150 \\
\hline
\end{tabular}

\section{4. 城市内满灾害的承灾体脆弱性分析}

根据所得数据情况, 选取受灾人口比例 (受灾人 口与常驻人口的比值) 作为城市内涝灾害承灾体的一 个单独指标, 开展承灾体脆弱性评估。

城市地区的人口数量会随着区域经济的发展和 社会结构的不断调整, 进而发生或快或慢的变化。由 于风险分析研究要描述的是不利事件在未来可能发 生的情景, 在研究承灾体脆弱性时涉及的人口数据时 也必须是案例区在未来某个时间段的常住人口数量 和受灾人口数量。鉴于基础地理数据的局限性, 选取 汕头市龙湖区作为案例区进行承灾体脆弱性评估和 灾情模拟。

\section{1. 城市常住人口预测}

关于人口数量的预测方法有很多, 例如: 指数增 长模型、Logistic 增长模型、自回归模型、灰色系统 模型、时间序列模型、神经网络模型、GEP 模型、 Leslie 模型等 ${ }^{[14-17]}$ 。考虑到所得实际数据类型, 本文 采用 Logistic 人口预测模型 ${ }^{[18]}$ 进行人口预测分析, 最 终得到案例区在未来几年的人口预测值 (见表 6)。

\section{2. 城市内港灾情模拟}

对于未来某时段内城市内涝灾害可能造成的受 灾人口模拟统计, 可以通过仿真模拟软件实现。本研 究在 VisioStudio 2008 环境下, 利用 $\mathrm{C} \#$ 编程语言和 ArcGIS 9.3 的 ArcEngine 二次开发组件, 建立了一套

表 6 案例区人口变化预测 (2014-2020 年)

\begin{tabular}{cccc}
\hline 年份 & 真实值 $($ 万 $)$ & 预测值 $($ 万 $)$ & 误差 $(\%)$ \\
\hline 2009 & 38.72 & 38.72 & 0 \\
2010 & 39.64 & 39.68 & 0.1 \\
2011 & 40.36 & 40.48 & 0.3 \\
2012 & 40.99 & 41.11 & 0.3 \\
2013 & 41.66 & 41.95 & 0.7 \\
\hline 2014 & $/$ & 43.67 & $/$ \\
2015 & $/$ & 45.23 & $/$ \\
2016 & $/$ & 47.85 & $/$ \\
2017 & $/$ & 50.51 & $/$ \\
2018 & $/$ & 54.06 & $/$ \\
2019 & $/$ & 57.24 & $/$ \\
2020 & $/$ & 61.36 & $/$ \\
\hline
\end{tabular}


Risk Analysis and Crisis Response in Big Data Era (RAC-16)

简单的城市内涝灾害模拟评估（CFDAS）系统，用 于模拟城市暴雨内涝积水淹没情景和受灾人口灾情 统计等。

CFDAS 系统利用 GIS 的空间分析技术, 首先对 已有的城市 DEM 数据和城市建筑物分布进行叠加和 修正处理, 形成城市建筑物高程图层; 其次, 根据河 流和城市街道的分布, 模拟了城市排水管道的分布情 况, 生成城市排水区域分布图; 再次, 根据降雨模型, 生成降雨图层; 然后, 将城市建筑物高程图层、城市 排水区域分布图层和降雨图层进行叠加计算, 生成不 同降雨条件下的淹没情况分布图; 最后, 在预设常住 人口平均分布在各个建筑物内的前提下, 统计出在不 同降雨淹没条件下的受灾人口分布情况。

基于表 5 拟定的条件, 假设在 2020 年案例区的 总人口为 61.36 万, CFDAS 系统便可以模拟出极端 气候条件下 (150mm 降雨) 的城市内涝积水和受灾 人口分布情况（参见图 6)。

\section{3. 城市内涝灾害的人口脆弱性评估}

为了便于政府、保险公司等公共部门的应用需 求, 我们以 1 年和 1 个国民经济规划阶段 (5 年) 为 时间约束单位, 进行不同降雨条件下的城市暴雨内涝 灾害受灾人口模拟和统计分析。

基于上述灾害模拟系统和人口预测, 就可以模拟 统计出案例区在未来几年不同降雨条件下的受灾人 口数据。根据 “最坏情景” 的评估原则, 我们通常会 模拟极端气候条件下的受灾情况 (参见表 7)。

由表 7 可以看出, 受灾人口统计样本数量非常 小。那么, 采用传统的统计方法就很难利用小样本找 对相应的函数并作出合理的人口脆弱性曲线。鉴于这 种信息不完备现象,我们可以利用信息扩散技术的函 数关系识别方法 ${ }^{[19,20]}$, 经过模糊推理, 给出降雨强度 与人口脆弱性指标之间的模糊关系, 进而计算出 “十
三五”期间案例区历年的暴雨内涝人口脆弱性变化向 量, 然后将这些脆弱性向量转为直观的曲线形式 (参 见图 3)。

\section{5. 城市暴雨内涝灾害风险的动态耦合}

自然灾害风险是致灾因子、承灾体、孕灾环境在 未来时段内相互作用可能产生的不利情景。因此, 在 计算城市内涝灾害风险时, 需利用综合风险评估模式 ${ }^{[20]}$ 将降雨 (致灾因子) 概率分布曲线和人口 (承灾体) 脆弱性曲线进行耦合, 其耦合计算的结果就是用来表 达某未来时段内暴雨内涝灾害的 “风险值”。

如果用 $\mathrm{R}_{\mathrm{e}}$ 表示城市暴雨内涝灾害 “风险”, $\mathrm{P}_{\mathrm{e}(\mathrm{x})}$ 表示降雨的概率分布函数, $\mathrm{F}_{\mathrm{e}(\mathrm{x})}$ 表示人口脆弱性函数, $\mathrm{x}$ 表示降雨量, $\mathrm{e}$ 表示年份, 则有:

$$
R_{e}=\int_{50}^{150} P_{e}(x) \cdot F_{e}(x) d x
$$

由公式 3 可知:

$P_{e}(x)=\exp \left(-\left(\frac{x-52.879}{104.73}\right)^{1.816}\right)$

(公式 5)

由上,城市暴雨内涝灾害风险的耦合计算方法可 用下图表示 (参见图 4)。

图 4 中的阴影部分即是由暴雨发生概率、人口脆 弱性和降雨强度共同约束下组成的内涝灾害 “ 风险 值”。“风险值” 的大小用降雨概率分布曲线、人口脆 弱性曲线以及降雨强度等三者所围成的阴影面积来 表示。

本研究中由于样本数据少, 人口脆弱性函数尚不 能用一个合适的连续函数表达, 因此当 $\mathrm{F}_{\mathrm{e}(\mathrm{x})}$ 可以用几 个离散值表示。以 2020 年为例, 当年发生城市内涝 灾害的风险值计算可以表示为:

表 7 案例区暴雨内涝灾害受灾人口模拟统计（2016-2020, 降雨条件：150mm）

\begin{tabular}{ccccc}
\hline 发展阶段 & 年份 & 常住人口 (万) & 受灾人口 (万) & 受灾人口比例 \\
\hline & 2016 & 47.85 & 7.17 & 0.150 \\
& 2017 & 50.51 & 7.29 & 0.144 \\
& 2018 & 54.06 & 8.02 & 0.148 \\
& 2019 & 57.24 & 8.55 & 0.148 \\
\hline
\end{tabular}


Risk Analysis and Crisis Response in Big Data Era (RAC-16)


图 3 案例区 “十三五” 期间的暴雨内涝灾害人口脆弱性曲线逐年变化图

$R_{2020}=\sum_{i=1}^{6}\left(\exp \left(-\left(\frac{x_{i}-52.879}{104.73}\right)^{1.816}\right) \times F_{2020}\left(x_{i}\right)\right)$

式中: $x_{\mathrm{i}}=[50,70,90,110,130,150]$,

$F_{2020}\left(x_{\mathrm{i}}\right)=[0.098,0.107,0.116,0.123,0.130,0.138]$
根据上述计算方法, 分别可以求得案例区在 “十 三五” 期间历年发生暴雨内涝灾害的 “风险值” (参 见图 5), 分别是 $\mathrm{R}_{2016}=0.3607, \mathrm{R}_{2017}=0.3612, \mathrm{R}_{2018}$ $=0.3543, \mathrm{R}_{2019}=0.3535, \mathrm{R}_{2020}=0.3493$ 。 
Risk Analysis and Crisis Response in Big Data Era (RAC-16)

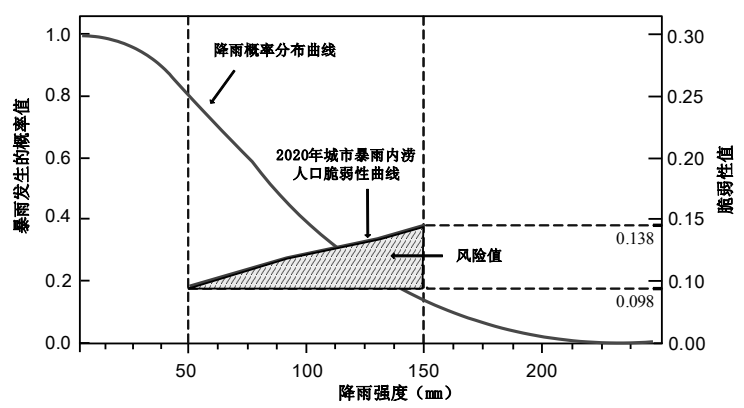

图 4 城市暴雨内涝灾害风险的耦合计算 (以 2020 年模拟数据为例)

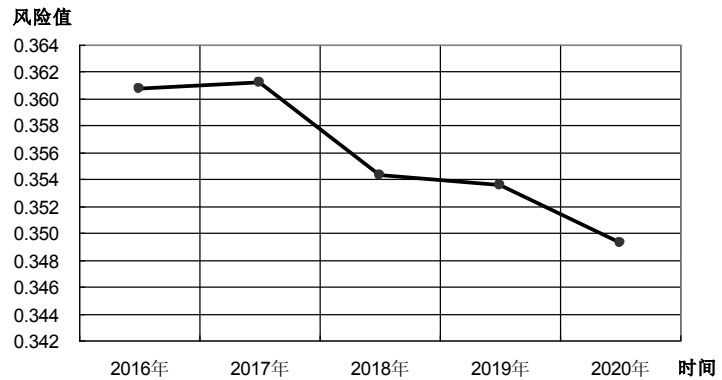

图 5 案例区 “十三五”期间城市暴雨内涝灾害风险趋势变化图

6. 城市内涝灾害动态风险的情景输出

根据城市暴雨内涝灾害风险的动态分析流程, 并 基于城市内涝灾害仿真系统的情景模拟, 结合对案例
区暴雨发生可能分析以及人口脆弱性的动态评估, 就 可以输出案例区在未来某时段内发生内涝灾害的动 态风险情景（参见表 8)。

表 8 案例区 “十三五” 期间发生城市暴雨内涝灾害风险的情景分析示意表

\begin{tabular}{|c|c|c|c|c|c|}
\hline \multirow[t]{2}{*}{ 年代 } & \multirow{2}{*}{ 风险值 } & \multicolumn{2}{|c|}{$\begin{array}{c}\text { 城市内港灾害情景 } 1 \\
\text { (50mm 降雨) }\end{array}$} & \multicolumn{2}{|c|}{$\begin{array}{c}\text { 城市内涝灾害情景 } 2 \\
\text { (150mm 降雨) }\end{array}$} \\
\hline & & 重现期 & 受灾人口 & 重现期 & 受灾人口 \\
\hline 2016 & 0.3607 & \multirow{5}{*}{1 年 } & 4.07 万 & \multirow{5}{*}{5 年 } & 7.17 万 \\
\hline 2017 & 0.3612 & & 4.32 万 & & 7.29 万 \\
\hline 2018 & 0.3543 & & 4.66 万 & & 8.02 万 \\
\hline 2019 & 0.3535 & & 4.91 万 & & 8.55 万 \\
\hline 2020 & 0.3493 & & 5.32 万 & & 9.08 万 \\
\hline
\end{tabular}

至此, 我们便可以分析认为案例区在 “十三五期 间”（2016-2020 年）的暴雨内涝灾害风险逐年递减, $50 \mathrm{~mm}$ 降雨发生的可能性最大, 重现期为 1 年; $150 \mathrm{~mm}$ 降雨发生的可能性较小, 重现期为 5 年。在 2016-2020 年间, 案例区遭受暴雨袭击后, 在梅溪和新津河沿岸
的大多数居民地将会受到内涝积水的影响, 随着降雨 量的增加, 内涝淹没范围会逐步向西部蔓延 (参见图 6)。到 2020 年, 发生 $50 \mathrm{~mm}$ 降雨时, 受灾人口约有 7.17 万人; 若发生 $150 \mathrm{~mm}$ 降雨, 受灾人口则达到 9.08 万左右。 
Risk Analysis and Crisis Response in Big Data Era (RAC-16)

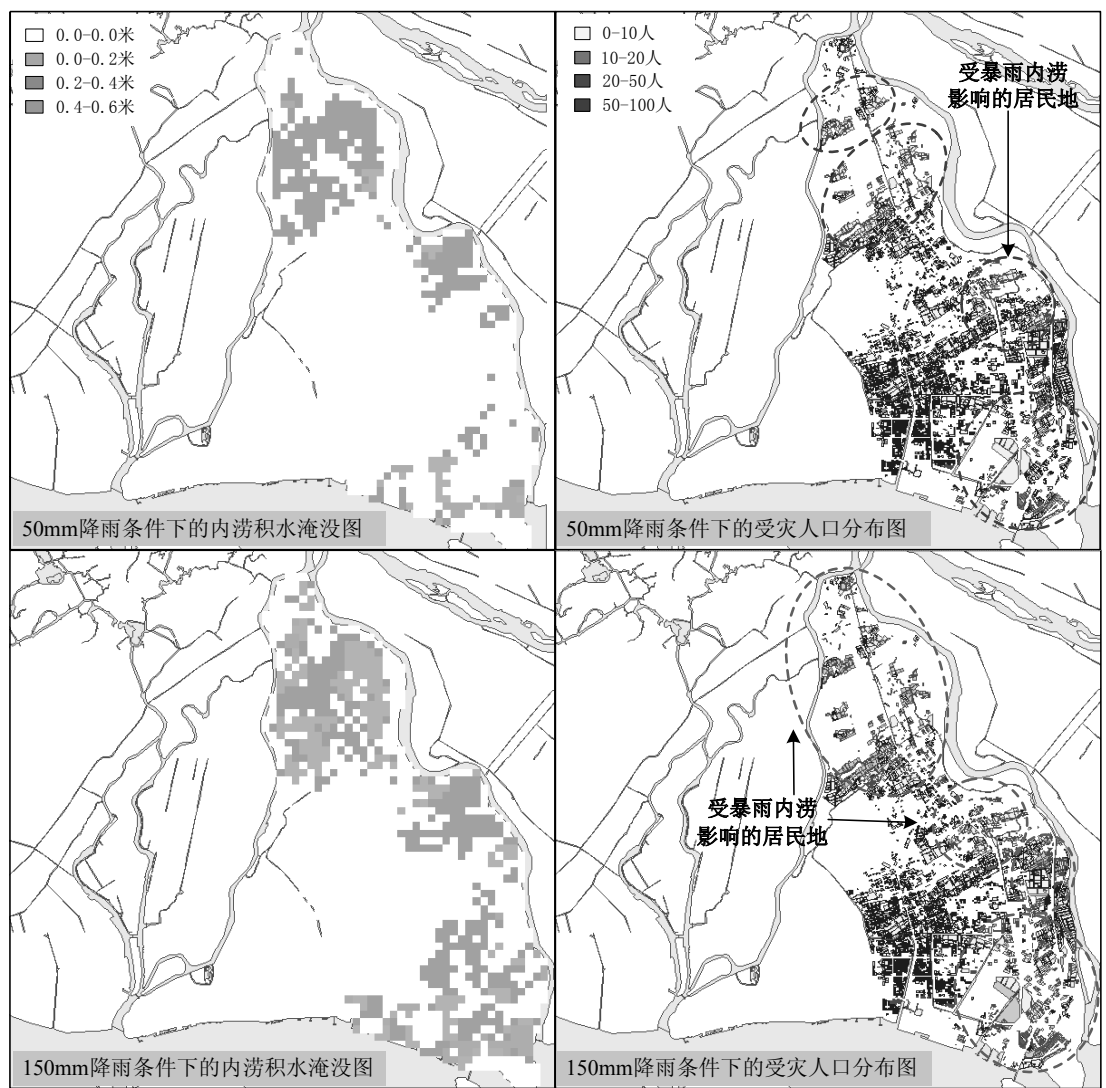

图 6 不同降雨条件下的城市暴雨内涝受灾情景图

在实际应用中, 可以根据基础数据的数量和精细 度对图 6 和表 8 中的数字信息和图像信息进行简化或 丰富, 从而在不同程度地满足政府公共部门开展灾害 风险管理工作的决策需求, 也可为保险公司制定保险 方案、厘定巨灾保险费率等提供参考。

\section{7. 结论讨论}

本研究将传统的参数估计统计方法、基于模糊计 算原理的信息扩散技术和地理信息空间模拟技术相 结合, 在遵从灾害风险的系统性、动态变化性等特征 的前提下, 利用综合风险评估模式, 探索性地分析了 未来几年城市暴雨内涝灾害风险的变化情况。虽然研 究结果可以较直观为相关部门提供决策支持, 但在研 究过程中还存在一些不足之处:
（一）基础数据的缺乏问题。例如, 在搭建城市 暴雨内涝灾害仿真模拟系统时, 因数据的缺失, 没有 考虑土壤下渗、地表蒸发、河流等因素以及水流动力 对内涝积水分布的影响, 模拟效果比较粗粘; 没有建 筑物和其它基础设施的结构数据和空间分布数据, 未 能开展除受灾人口外其他承灾体的脆弱性分析; 进行 人口脆弱性评估时, 在无法获得具体土地利用规划数 据和居民实际分布情况的前提下, 只能假设未来案例 区的居民地分布情况不变、城市居民均匀地分布在居 民地中。

（二）分析方法的选择问题。受模型和数据的影 响, 只能利用历史数据和传统的统计方法计算暴雨在 未来时段发生的可能性; 同样由于所获数据的局限 性, 暂时只能用简单的人口模型进行人口变化预测。 
Risk Analysis and Crisis Response in Big Data Era (RAC-16)

本文提出的城市内涝灾害的动态风险分析流程 以及相关分析方法, 存在一定的狭义性和局限性, 均 需在未来研究中开展进一步的探索和完善。

\section{致谢}

本研究得到了国家社科基金青年项目“生态文明 视角下环境突发事件应急管理问题研究” (13CGL134) 的资助。

\section{参考文献}

[1] Allianz. Allianz Risk Pulse-Focus: Floods. Germany, 2011, 1-6. https://www.allianz.com/ media/responsibility/documents/risk pulse floods en.pdf

[2] 李伟峰,陈求稳,毛劲乔. 北京奥运村洪水淹没风 险模型研究. 科学通报, 2009, 54(3): 321-328

[3] 景垠娜,尹占娥,殷杰等. 基于 GIS 的上海浦东新 区暴雨内涝灾害危险性分析. 灾害学, 2010, 25(2): $58-63$

[4] Jiang Weiguo, Deng Lei, Chen Luyao, et.al. Risk assessment and validation of flood disaster based on fuzzy mathematics. Progress in Natural Science, 2009, (19): 1419-1425

[5] Camarasa-Belmonte A.M. Soriano-Garc í a J. Flood risk assessment and mapping in peri-urban mediterranean environments using hydrogeomorphology application to ephemeral streams in the Valencia region. Landscape Urban Planning, 2012, 104(2): 189-200

[6] Huttenlau M., Stotter J., and Stiefelmeyer H. Risk-based damage potential and loss estimation of extreme flooding scenarios in the Austrian Federal Province of Tyrol. Natural Hazards and Earth System Sciences，2010，(10): 2451-2473

[7] 尹占娥,许世远,殷杰. 基于小尺度的城市暴雨内 涝灾害情景模拟与风险评估. 地理学报, 2010, 65(5): 553-562

[8] Rahman Khatibi. Evolutionary systemic modelling of practices on flood risk. Journal of Hydrology, 2011, (401): 36-52

[9] 黄崇福. 综合风险评估的一个基本模式. 应用基 础与工程科学学报, 2008, 16(3): 371-381

[10] 中国气象局国家气象信息中心.中国气象科学数 据共享服务网 [ER/OL]. http://cdc.cma.gov.cn, 2014-11-1

[11] IPCC. IPCC Fourth Assessment Report: Climate Change 2007_working Group II Report: Impacts, Adaption and Vulnerability. Cambridge: Cambridge University Press, 2007: 478-486

[12] Prudhomme C., Wilby R.L., Crooks S., et.al Scenario-neutral approach to climate change impact studies: Application to flood risk. Journal of Hydrology， 2010， (390): 198-209

[13] Satish Bastola, Conor Murphy, John Sweeney. The sensitivity of fluvial flood risk in Irish catchments to the range of IPCC AR4 climate change scenarios. Science of the Total Environment, 2011, (409): 5403-5415

[14] 陈强. 人口系统模型及人口状况分析. 南京理工 大学, 2004, 30-42

[15] 刘萌伟,黎夏,刘涛. 基于基因表达式编程的人口 预测模型. 中山大学学报: 自然科学版, 2010, 49(6): $115-120$

[16] 刘赛瑜,黄国祥. 中国离散型人口模型分析. 科 学技术与工程，2010，10(21)：5225-5229

[17] 雅科夫.Y.海姆斯. 风险建模、评估和管理(第二 版). 西安: 西安交通大学出版社, 2007, 155-180

[18] 阎慧鉌. Logistic 模型在人口预测中的应用. 大 连工业大学学报，2008，27(4)：333-335

[19] Xilei Pang. Dynamic Vulnerability Analysis of Population for Flood Disaster in Urban Area. Journal of Risk Analysis and Crisis Response, 2013, 3(4):166-174

[20] 黄崇福. 自然灾害风险分析与管理. 北京: 科学 出版社, 2012 